\title{
Managing earwax in primary care: efficacy of self-treatment using a bulb syringe
}

\author{
Richard Coppin, Dorothy Wicke and Paul Little
}

\begin{abstract}
Background

Irrigating ears to remove wax is a time-consuming procedure in UK primary care. In many other countries bulb syringes are used for self-clearance of earwax but evidence of their effectiveness is lacking.

Aim

To compare the effectiveness of self-treatment bulb syringes with routine care.

Design of study

Open, randomised, controlled trial.

Setting

Seven practices in Hampshire, UK.

\section{Method}

Participants were 237 patients attending their GP or practice nurse with symptomatic occluding earwax. A further 128 patients did not want to be part of the randomisation but allowed their data to be analysed. Patients randomised to intervention $(n=118)$ were given ear drops, a bulb syringe, and instructions on its use. Patients in the control group $(n=119)$ received ear drops, followed by ear irrigation by the GP or practice nurse. Main outcome measures were symptoms (on a 7-point scale), wax clearance, need for further treatment, and the acceptability of treatment.

\section{Results}

Comparing patients using the bulb syringe with those treated with conventional irrigation, the change in mean symptom score was $\tilde{A} 0.81$ and $\tilde{A} 1.26$ respectively (difference $\tilde{A} 0.45,95 \%$ confidence interval $[\mathrm{Cl}]=\tilde{A} 0.11$ to $\tilde{A} 0.79$ ) and, regarding the proportion requiring no further irrigation, $51 \%$ and $69 \%$ respectively. Although irrigation was preferred by more patients, most patients using the bulb syringe would use it again (75\% versus $100 \%)$ and were satisfied with treatment (71\% versus $99 \%)$.

Conclusions

Advising patients with ears blocked by wax to try bulb syringing before irrigation is effective and acceptable, and could significantly reduce the use of NHS

resources.
\end{abstract}

Keywords

Adult; cerumen; ear canal; family practice; irrigation.

\section{INTRODUCTION}

Ears blocked by wax can be uncomfortable and irritating, and those suffering such symptoms often seek rapid relief. In the UK, managing symptomatic earwax is a traditional and significant demand on primary care services. A survey of GPs suggested that out of a population of 650000,44000 ears were syringed annually. ${ }^{3}$ Although this task was previously within the remit of GPs, clearance of wax is now mostly carried out by nurses using electronic irrigators rather than traditional piston syringes. ${ }^{4}$

In the US and many European countries $E$ but not in the UK $E$ inexpensive, plastic, bulb syringes for irrigating the ear to clear wax are commonly available over the counter at pharmacies. There are no data on the use of bulb syringes in the international literature, perhaps because they are traditional devices in the countries where they are used and have thus never required formal evaluation. If they are a safe and effective way of self-managing symptomatic wax, their use could be encouraged with the potential to reduce time spent by health professionals on this common activity, and to give patients greater control of the problem.

UK GPs and patients appear receptive to the idea of self-help. ${ }^{4}$ In a pilot observational study by the current authors, 50 primary care patients were offered self-use of a bulb syringe as an alternative to routine ear syringing. Median duration of use was 3 days and most participants reported that the method was acceptable.

R Coppin, GP, BMedSci, DRCOG; D Wicke, RGN, research nurse, The Surgery, Overton; P Little, MSc, MD, MRCP, FRCGP, DLSHTM, professor of primary care research, Aldermoor Health Centre, Southampton.

Address for correspondence

Dr Richard Coppin, The Surgery, Station Road, Overton,

RG25 3DU. E-mail: richard.coppin@nhs.net

Submitted: 10 May 2007; Editor's response: 20 July 2007; final acceptance: 26 September 2007.

(c)British Journal of General Practice 2008; 58: 44-49.

DOI: 10.3399/bjgp08X263811 
The current study aimed to investigate a sample of patients with symptomatic occluding earwax to compare the effectiveness of self-use bulb syringes with routine electronic irrigation carried out by practice nurses.

\section{METHOD}

\section{Intervention}

Bulb syringes are available from a number of manufacturers and in varying sizes. The $1 \mathrm{oz}$ $(25 \mathrm{ml})$ size (bulk cost each: less than £0.50, €0.8, US\$1) appeared to be the most commonly available in the US and in Germany, and was the size chosen for this study.

With self-use of the bulb, increasing the pressure of the water jet is painful and discourages excessive force. However, compared with the metal tip of a traditional syringe or the plastic tip of an electronic ear irrigator, the tip of a bulb syringe is much softer and more flexible. The nozzle of the bulb increases in diameter away from the tip preventing over-penetration into the canal. The researchers devised an information sheet for study participants based on those from the German manufacturer and US health information sources that warned not to use excessive force or to push the tip into the ear canal. It also gave advice on how to clean the device with a view to re-use.

\section{Participants}

Seven general practices in Hampshire in the south of England agreed to participate. Researchers trained the practice nurses at each practice on use of the protocol and ear blockage assessment. Adult patients consulting a GP or practice nurse with symptoms suggestive of occluding earwax (itching, sensation of blockage, and reduced hearing) were invited to participate. Patients were given a verbal explanation of the protocol and a copy of the patient information sheet. Patients were assessed for eligibility (symptoms and at least one ear canal occluded with wax), and were given

\section{How this fits in}

Irrigating ears to remove wax is a time-consuming procedure in UK primary care. In many other countries bulb syringes are used for selfclearance of earwax. This study suggests that bulb syringes are effective. Advising patients with symptomatic wax to use a bulb syringe as firstline treatment could reduce the need for ear syringing in primary care. the opportunity to ask questions. To enable assessment of the generalisability of results, patients were given the choice of three participation options:

- Full consent (full consent to randomisation);

- Notes-search only (non-participation but consenting to a later notes search);

- Declining (non-participation).

Those not consenting to full participation in the trial were offered usual treatment of ear drops followed by ear irrigation by the practice nurse using an electronic irrigator. Patients who had fully consented and those consenting to a notes-search only were asked to complete a short questionnaire to record baseline data.

Patients who fully consented were randomised. They were each given an envelope that had previously been randomised using random number tables by a member of the team not involved in data collection or recruitment. Envelopes were numbered and contained a questionnaire to be completed after 1 week and a box containing either ear drops, a bulb syringe, and instructions on its use; or ear drops and a roll of card of similar weight to the bulb and instructions on usual treatment.

Sealed envelopes were used in consultations for logistic reasons: to ensure that the GP or nurse consulting with the patient immediately had all that was required for intervention (the bulbs and instructions), and to avoid the delay of phoning a randomisation line. Use of envelopes was audited and there was no evidence from the baseline tables that there was selective recruitment and, therefore, no evidence of potential confounding. Patients were not trained in bulb use, and researchers did not perform intrusive assessment of compliance (which is unlikely to occur in practice). Nurses reported no case of patients not using the bulbs at follow-up.

For initial wax softening, sodium bicarbonate drops were chosen as they are commonly used and it is not known which wax-softening drops are most effective. ${ }^{5}$ The instructions advised use of drops for at least 2 days before irrigation. If allocated to usual treatment, and if the patient had applied drops already, irrigation could be carried out during the appointment, otherwise it was arranged a few days later. All patients in both groups were given a second study appointment 1-2 weeks later, as the pilot study revealed that patients finished using the bulb after 1 week.

At the second appointment patients handed in the second questionnaire. The practice nurse then carried out ear examination to assess clearance of 


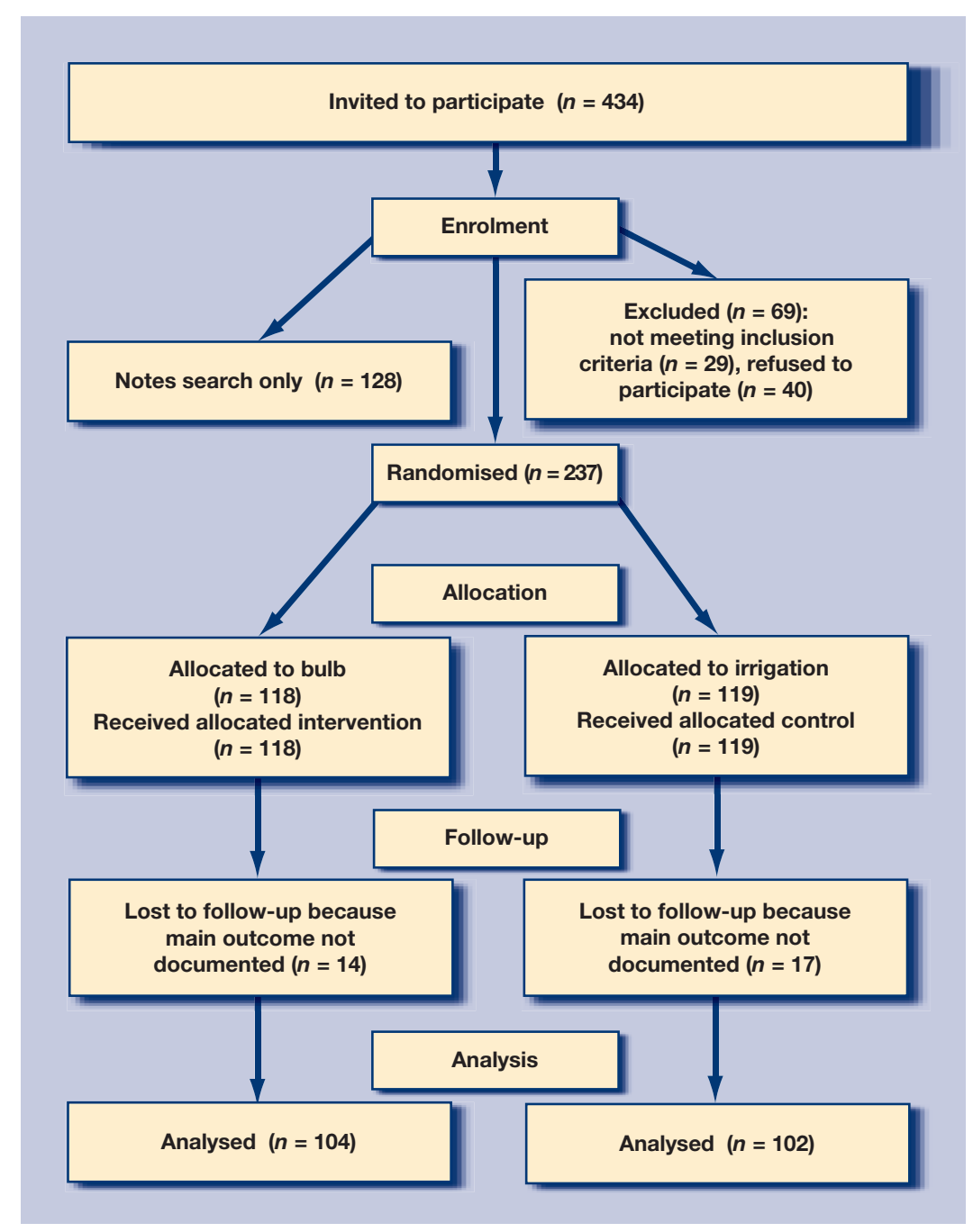

Figure 1. Participant flowchart for those consenting to full participation. wax and unwanted effects of treatment, such as infection or trauma. If significant blockage remained, further irrigation was given in line with normal clinical practice.

\section{Main outcome measures}

Overall symptoms for one or both ears (discomfort, itching, hearing, and overall problems) and the acceptability of treatment (convenience, control, and satisfaction) were assessed using questions validated in the pilot study and recorded on a 7point scale. Based on the results of factor analysis, a measure of the four symptom items showed high internal reliability (Cronbach's $\alpha>0.80$, that is, in the optimal range). The four symptom items were combined to create a symptom score, scaled from 0 to 6 . The score had construct validity: patients' scores (blind to assessment of blockage) related almost linearly to the observed blockage by nurses (who did not see patients' ratings). Thus, for patients with no significant obstruction ( 0 or 1 in the score shown below), one ear with significant obstruction, and two ears with significant obstruction, mean symptom scores were 0.78 , 1.81 , and 2.56 respectively (test for trend $P<0.001$ ).

Among patients where there was no significant wax at follow-up (the group where a change in symptom score would be expected) the standardised response mean was 1.45 (1.61/1.11), that is, very sensitive to change.

Wax obstruction was measured by a practice nurse using a previously described 4-point obstruction score $^{6}$ which had been used in the pilot study:

- $0=$ no or minimal wax with tympanic membrane fully visible;

- 1 = minor amount of wax with tympanic membrane essentially visible;

- 2 = moderate amount of wax with tympanic membrane partially obscured; and

- 3 = complete occlusion of tympanic membrane.

In the analysis, scores of 0 or 1 were combined to indicate a clinically 'clear' ear. In the routine setting of the study practices it was not logistically possible to achieve blinded assessment of observed wax clearance.

Initial assessment was carried out by a nurse ( $n$ $=230)$ or GP $(n=7)$, and all irrigation and follow-up assessments were carried out by a nurse who may or may not have carried out the pre-treatment assessment (28\% were by the same nurse). Nurses recorded the need for further irrigation (after initial bulb/irrigation) based on normal clinical practice within 6 weeks from randomisation, unwanted effects of treatment, and rates and reasons for non-participation or non-compliance.

\section{Sample size}

Principal outcomes were reported symptoms and wax clearance. Key secondary outcomes were the need for further irrigation and acceptability of treatment. Observed wax clearance was the limiting variable in the sample-size calculation (for $\alpha=0.05$ and power $=80 \%$ ). Based on the pilot study, assuming $75 \%$ cleared wax using the bulb and $90 \%$ with syringing (that is, a $15 \%$ absolute difference between groups), it was estimated that 100 patients were needed in each group; or allowing for $15 \%$ loss to follow-up, 236 in total. This sample size allowed detection of a 0.4 standard deviation (SD) difference in continuous outcomes (symptom score). It was estimated from piloting that this would easily allow assessment of whether symptoms were rated a slight problem rather than a moderate problem.

\section{Analysis}


Table 1. Participants' baseline data.

\begin{tabular}{lcccc} 
& Randomisation groups & Non-randomisation groups \\
\hline & $\begin{array}{c}\text { Bulb } \\
(n=118)\end{array}$ & $\begin{array}{c}\text { Irrigation } \\
(n=119)\end{array}$ & $\begin{array}{c}\text { Notes search } \\
\text { only }(n=128)\end{array}$ & $\begin{array}{c}\text { Declined/excluded } \\
(n=69)\end{array}$ \\
\hline $\begin{array}{l}\text { Mean (SD) symptom scorec at baseline, } \\
n=205\end{array}$ & 2.37 (SD 1.44) & 2.41 (SD 0.90) & - & - \\
\hline $\begin{array}{l}\text { Right ear completely } \\
\text { obstructed with wax, } n(\%)\end{array}$ & $73 / 116(63)$ & $72 / 116(62)$ & $82 / 128(64)$ & $E$ \\
\hline $\begin{array}{l}\text { Left ear completely } \\
\text { obstructed with wax, } n(\%)\end{array}$ & $78 / 116(67)$ & $79 / 114(69)$ & $84 / 128(66)$ & - \\
\hline $\begin{array}{l}\text { Male, } n(\%) \\
\text { Mean (SD) age, years }\end{array}$ & $78 / 118(66)$ & $74 / 117(63)$ & $74 / 128(58)$ & $36 / 69(52)$ \\
\hline
\end{tabular}

cSymptom score: $0=$ no symptom to $6=$ severe.

Groups were compared by intention-to-treat analysis using analysis of covariance and the KruskalÃWallis test for continuous outcomes (symptoms score) and $\chi^{4}$ test or Fisher's Exact test (for low cell counts) for dichotomous outcomes, such as wax clearance. The trial was designed as an individually randomised trial: it was not cluster randomised. As expected when using well-defined interventions, no clustering was found by practice or nurse. Allowing for clustering in the analysis made no difference to the inferences. As such, the results are presented with no allowance for clustering.

\section{RESULTS}

A total of 434 patients were invited to participate between March and October 2004. Of these, 69 (16\%) declined or were excluded. Of those remaining, 237 were randomised and a further 128 , although not wishing to be randomised, agreed to their notes being searched. No patient who was offered a bulb declined to use it, or reported not using it. The assessment of wax at follow-up was available for 206 patients (88\%; Figure 1) .

Baseline data of age, sex, and ear obstruction were similar in the randomised and nonrandomised groups (Table 1). Most patients returned for follow-up within 2 weeks (on average at $8-10$ days). This did not differ significantly between groups: mean follow-up of bulb group was 10 days, and for the irrigation group was 11 days. Controlling for time of follow-up made no difference to estimates or inferences. There was a minimal difference in the number of days that drops were used for softening the wax in either group (bulb group mean $=3.5$ days; irrigation group mean $=3.6$ days).

Patients using a bulb achieved a reduction in mean symptom score (scaled 0 to 6 ) of $64 \%$ of the irrigation group ( $\tilde{A} 0.81$ versus $\tilde{A} 1.26$, difference $\tilde{A} 0.45 ; 95 \%$ confidence interval $[\mathrm{Cl}]=\tilde{A} 0.11$ to Ão.79; Table 2). A total $48 \%$ of patients using the

\section{Table 2. Symptoms and satisfaction: comparison between group for changes in means (SD of change) and proportions after 1 week.}

\begin{tabular}{lcccc} 
& Bulb & Irrigation & $\begin{array}{c}\text { Difference between } \\
\text { groups (95\% Cl) }\end{array}$ & $P$-value \\
\hline $\begin{array}{l}\text { Mean (SD) change in symptom scorec } \\
\text { from baseline }\end{array}$ & $\tilde{A} 0.81(1.44)$ & $\tilde{A} 1.26(1.15)$ & $\tilde{A} 0.45(\tilde{A} 0.11$ to $\tilde{A} 0.79)$ & $0.01(0.02)^{d}$ \\
\hline $\begin{array}{l}\text { Treatment discomfort } \\
\text { (slight or more), } n \text { (\%) }\end{array}$ & $43 / 110(39)$ & $35 / 108(32)$ & $7 \%(\tilde{A} 6$ to $19 \%)$ & 0.30 \\
\hline $\begin{array}{l}\text { Treatment dizziness } \\
\text { (slight or more), } n \text { (\%) }\end{array}$ & $14 / 110(13)$ & $14 / 108(13)$ & $0 \%(\tilde{A} 9$ to $9 \%)$ & 0.96 \\
\hline $\begin{array}{l}\text { Treatment convenient } \\
\text { (agreed slightly or more), } n(\%)\end{array}$ & $84 / 110(76)$ & $95 / 105(90)$ & $14 \%(4$ to $24 \%)$ & $<0.01$ \\
\hline $\begin{array}{l}\text { Satisfied with treatment, } n(\%) \\
\text { (agreed slightly or more) }\end{array}$ & $78 / 110(71)$ & $105 / 106(99)$ & $28 \%(19$ to $29 \%)$ & $<0.001$ \\
\hline $\begin{array}{l}\text { Use same treatment again, } n(\%) \\
\text { (agreed slightly or more) }\end{array}$ & $82 / 110(75)$ & $106 / 106(100)$ & $25 \%(17$ to $25 \%)$ & $<0.001$ \\
\hline
\end{tabular}

cSymptom score: 0 = no symptom to 6 = severe. ${ }^{a}$ Kruskal-Wallis test. 


\begin{tabular}{|c|c|c|c|c|}
\hline & Bulb, $n(\%)$ & Irrigation, $n(\%)$ & $\begin{array}{l}\text { Difference between } \\
\text { groups }(95 \% \mathrm{Cl})\end{array}$ & $P$-value \\
\hline Wax clearance (obstruction score 0 or 1 ) & $50 / 104(48)$ & 64/102 (63) & $15 \%$ (1 to $28 \%)$ & 0.03 \\
\hline $\begin{array}{l}\text { Requires no further clearance } \\
\text { (based on normal clinical practice) }\end{array}$ & $51 / 100(51)$ & $66 / 95(69)$ & $18 \%$ (5 to $32 \%)$ & $<0.01$ \\
\hline Infection & $1 / 97(1)$ & $1 / 93(1)$ & $0 \%(\tilde{3}$ to $3 \%)$ & $1.00^{c}$ \\
\hline Perforation & $1 / 97(1)$ & 1/94 (1) & $0 \%(\tilde{A} 3$ to $3 \%)$ & $1.00^{\circ}$ \\
\hline Signs of trauma & $1 / 97(1)$ & 1/94 (1) & $0 \%(\tilde{A} 3$ to $3 \%)$ & $1.00^{c}$ \\
\hline
\end{tabular}

bulb achieved adequate wax clearance (obstruction score 0 or 1) compared with $63 \%$ in the irrigation group (difference $15 \%, 95 \% \mathrm{Cl}=1$ to 28; Table 3). However, following clinical assessment by the practice nurse, the number of patients needing re-irrigation was 49 out of 100 (49\%) patients in the bulb group, and 29 out of 95 (31\%) patients in the irrigation group. Thus, $51 \%$ (95\% Cl $=41$ to 61$)$ of patients given a bulb did not require their ears to be irrigated by the practice nurse.

Differences in rates of complications documented by practice nurses were minimal. In the bulb and irrigation groups there were low incidences of possible complications: infection ( $1 \%$ versus $1 \%$ respectively), perforation ( $1 \%$ versus $1 \%)$, or signs of trauma (1\% versus $1 \%)$; similar levels of treatment discomfort were identified in the bulb and irrigation groups (39\% versus $32 \%$ ). Most of the adverse 'trauma' events reported were mild (slight localised erythema). One patient in the irrigation group was noted to have bilateral otitis externa and, of the two patients noted to have a perforation, subsequent assessment showed one to have old scarring of the tympanic membrane and the other had pre-existing cholesteotoma which was confirmed following specialist referral.

Although irrigation was preferred by more patients, most patients using the bulb syringe would use it again (bulb $75 \%$ versus irrigation $100 \%$ ) and were satisfied with treatment (bulb $71 \%$ versus irrigation 99\%).

\section{DISCUSSION}

\section{Summary of main findings}

At least half of patients given a bulb and simple instructions did not require further irrigation; both methods of treatment (earwax removal with a bulb syringe and with traditional irrigation) appear to have a similar safety profile.

\section{Comparison with existing literature}

To the authors' knowledge, this is the first randomised trial to assess the effectiveness of a simple pragmatic approach to bulb syringing.

\section{Strengths and limitations of the study}

A simple low-resource approach (providing the bulb and simple instructions) was used with no training and no assessment of compliance. This study was sufficiently powered to demonstrate a meaningful difference in the main outcomes. Recruitment bias was minimised as a high proportion of eligible patients were either recruited or allowed researchers access to their data. Dropout rates were low.

Ideally, changes in patient-reported symptoms should have been confirmed by blinded assessment of wax clearance, but performing blinded independent visual assessment was not possible for practical reasons. However, unblinded wax clearance provides very useful corroborative evidence for the main outcome measure: a patientcentred report of symptoms. Patient reporting of symptoms reflected the effect of the intervention on the presenting symptoms, was reliable, valid, and sensitive to change, and showed very similar effect sizes to wax clearance. The bulb had approximately two-thirds of the effect of irrigation. The effect on symptoms and wax clearance was also corroborated by the $75 \%$ of patients who would be happy to use bulbs as a first-line treatment.

This study reports the need for further treatment which was assessed and given by the practice nurses who managed these patients. Again, this assessment could not be blinded, but it is nevertheless legitimate as it represents the further use of resources that are likely to occur in everyday practice.

Regarding the safety of bulb syringes, reported treatment discomfort was greater (but not significantly so) in the bulb syringe group. Reports of possible trauma in 6 patients receiving either irrigation or bulb syringe were made at follow-up, and these were either mild or simply revealed pre- 
existing problems on further investigation.

There are no published data on the safety of bulb syringes or traditional syringing with which to compare these results. However, there have been safety concerns about irrigation of the external ear canal, particularly using traditional piston syringes.3.7.8 Over-the-counter availability of bulb syringes in the US and some European countries implies a lack of major safety concerns. The US Food and Drug Administration Center for Devices and Radiological Health has maintained an adverseevent register for medical devices since the early 1990s. A search of the database in September 2007 found no reports of adverse events from self-use of bulb syringes.

\section{Implications for clinical practice and \\ future research}

This study shows that although first-line treatment by irrigation carried out by a practice nurse is more effective, a third of those patients will probably have to return a second time for further irrigation. This compares with half of patients using a bulb syringe. Based on these rates, a policy of offering bulbs as an initial alternative to nurse irrigation would result in a worthwhile reduction in the number of irrigations performed.

A potential impediment to the implementation of this in the UK is the current limited availability of bulb syringes. However, there is no reason why pharmacies and practices should not be able to order stocks of bulbs for their patients to use.

This study suggests that it is probably reasonable to advise patients with blocked ears to try bulb syringing. This could significantly reduce the demand for routine ear syringing, and give patients more control of this common problem. Assessment of the long-term impact of self-treatment with bulb syringes on patients and on service costs requires further research.

\section{Funding body}

The study was funded by the Royal College of General Practitioners Scientific Foundation Board (reference SFB/2003/32) and supported by the UK Department of Health Support for Science funding

\section{Ethical approval}

Ethical approval was granted by the North and Mid Hampshire Local Research Ethics Committee, 3 September 2003 (03/A/057). Trial registration (ISRCTN71172551) was assigned to this study

\section{Competing interests}

The authors have stated that there are none

\section{Acknowledgements}

We are grateful to the members of the North Hampshire Primary Care Research Club for originating the research idea and for their subsequent support; Albert Hohlkorper $\mathrm{GmbH}$, Hemer, Germany for supplying the bulb syringes; the study practices for agreeing to participate; the patients and practice nurses for carrying out the study; and to Sally Bown for clerical support and data entry

\section{Discuss this article}

Contribute and read comments about this article on the Discussion Forum: http://www.rcgp.org.uk/bjgp-discuss

\section{REFERENCES}

1. Sharp JF, Wilson JA, Ross L, Barr-Hamilton RM. Ear wax removal: a survey of current practice. BMJ 1990; 301(6763): 1251-1253.

2. Coppin R, Wicke D, Mehta R, Little P. Management of earwax in primary care - postal survey of UK GPs and practice nurses. Fam Pract 2004; 21(4): 413-414.

3. Hand C, Harvey I. The effectiveness of topical preparations for the treatment of earwax: a systematic review. Br J Gen Pract 2004; 54(508): 862-867.

4. Sullivan RF. Video otoscopy-based scale of cerumen accumulation/EAC obstruction, 1995. www.rcsullivan.com/www/vocerum.htm (accessed 9 Nov 2007).

5. Blake P, Matthews R, Hornibrook J. When not to syringe an ear. NZ Med J 1998; 111(1077): 422-424.

6. Dinsdale RC, Roland PS, Manning SC, Meyerhoff WL. Catastrophic 